\title{
SÍNTESIS EVOLUTIVA DE INVESTIGACIONES EN ENSENANZA DE CIENCIAS
}

\author{
PESSOA DE CARVALHO, A.M. (Coordinadora), LABURU, C.E., DA SILVA, D., F, MORTIMER, E., \\ GARRIDO, E., TRIVELATO, J., BECHARA, L., ORIOSVALDO DE MOURA, M., GONÇAVES, M.E.R., \\ SANTOS, M., TEIXEIRA, O.P.B., RIBEIRO DO VALLE FILHO, M., DE CASTRO, R., ITACARAMBI, R.
}

Faculdade de Educaçāo. Universidade de Săo Paulo. Avda. da Universidade, 308. 05508 São Paulo - SP. Tf. 011-815.02.97. Fax -11-815.42.72. BITNET AMPDCARV BRUSP.

\section{SUMMARY}

In this article, the working team, linked to Sao Paulo University, analizes the works of research that it is presently carrying out and displays its projects for the future.

The works of research are made under two complementary lines: on the one hand, the thought in children and adolescents and, on the other, the evaluation of teaching methodologies embodying results of the first line of research in their design that are based on constructivist proposals.

Lo que proponemos en este trabajo es, en primer lugar, analizar críticamente la producción de nuestro grupo, presentando nuestras opciones y sus consecuencias y, en un segundo momento, mostrar los futuros planes que intentamos delinear.

Somos un grupo de profesores de escuela secundaria y universitaria, vinculados a la Faculdade de Educação de la Universidade de São Paulo, por mediación de sus dos programas de postgrado: el de postgrado en Educación (maestría y doctorado) y el de postgrado en Enseñanza de Ciencias, modalidad Física, éste en convenio con el Instituto de Física.

El grupo estableció dos líneas de investigación. Una que acompaña el raciocinio de los niños y adolescentes, y que estudia el desarrollo de los conceptos de las Ciencias (Física, Química, Biología y Matemáticas), principalmente de aquéllos que se enseñan en el $1 \%$ y $2^{\circ}$ grados; ${ }^{(1)}$ y otra que comprueba, en el aula, con carácter experimental, metodologías de enseñanza basadas en presupuestos constructivistas.
Estas dos líneas de investigación están muy relacionadas pues, tal y como señala Coll (1983): «... Ios contenidos escolares poseen un aito grado de especificidad y, consecuentemente, desconocemos completamente la forma por la cual los alumnos construyen progresivamente. Por lo tanto, será necesario conocer, con el máximo de detalles, el camino que el alumno sigue hacia la construcción de estos conocimientos específicos si deseamos, de hecho, lograr una adecuacion entre contenidos escolares y niveles de construcción psicogenética. Asimismo, convendrá conocer los procedimientos por los cuales el alumno se apropia progresivamente de estos contenidos, si deseamos intervenir eficazmente en su adquisición.»

De esta forma, nuestros trabajos en psicogénesis pretenden construir un conocimiento específico indispensable para comprobar la hipótesis de la necesidad de conocer el desarrollo lógico-causal de los conceptos, con el fin de elaborar la enseñanza de estos conceptos para el $1^{\circ}$ y $2^{\circ}$ grados. Estos conocimientos sirven como base no sólo para entender el raciocinio de nuestros alumnos, sino 
para que creemos situaciones desequilibradoras y reequilibradoras en la construcción de nuestras enseñanzas.

\section{ANÁLISIS CRÍTICO DE LOS TRABAJOS REALIZADOS}

En la primera línea, en la cual procuramos conocer cómo nuestros alumnos construyen los conceptos que la escuela deberá enseñar y cómo ellos explican las relaciones causales de los fenómenos presentados, la metodología de investigación que se utiliza es la entrevista clínica, pues ella nos of rece mayores garantías para diagnosticar el pensamiento del alumno. Realizamos nueve trabajos entre 1982 y 1990 (Anexo). Si se hace un análisis crítico del conjunto de esos trabajos, podemos distinguir dos puntos importantes: el primero es la interrelación entre la piscogénesis y la historia de la ciencia. Sentimos, en varios trabajos, lat necesidad de intentar entender, además, el desarrollo histórico del concepto, es decir, buscar el «flash» de esa evolución conceptual que caracterizó no sólo los puntos de ruptura, sino principalmente, los raciocinios utilizados por los científicos al sistematizar, cada ve $z$ con mayor exactitud, los conceptos que estudiábamos a cada momento (Piaget y García 1982, Gagliardi 1988, Gohau 1987, Lacombe 1987).

Tenemos conciencia de que no investigamos la historia de la ciencia con todas las retaciones socio-culturales de cadá época, sino que buscamos la dinámica del desarrollo del conocimiento científico. Buscamos, en la historia de la ciencia, las relaciones concebidas por los distintos científicos entre los varios conceptos, las experiencias que resultaron importantes en este desarrollo conceptual $y$, sobre todo, la evolución de las explicaciones causaies (Halbwach 1977).

El segundo punto importante que señalamos en la revisión crítica de los trabajos, fue la modificación en la elección de la muestra y en el análisis de los datos obtenidos a través de las entrevistas.

En los primeros trabajos (sobre visión, velocidad y aceleración), quizá bajo la influencia de las investigaciones con conceptos espontáneos, los alumnos seleccionados para la muestra eran aquéllos que frecuentaban $5^{\circ}$ y $8^{\prime \prime}$ curso del primer grado y $1^{\circ} \circ 2^{\circ}$ curso del $2^{\circ}$ grado ${ }^{\text {(2). }}$. Los análisis de los datos se elaboraron de forma horizontal, es decir, intentamos averiguar cómo cada grupo de alumnos respondía a cada fenómeno $\mathrm{y}$, a continuación, comparamos las respuestas entre los grupos.

Fn los demás trabajos (cantidad de movimiento, velocidad angular, modelo corpuscular, centro de masa y campo), elegimos una muestra de alumnos cuyas edades Iban de los 7 a los 14-15 años y que cursaban entre $1^{\text {g }}$ y $8^{\circ}$ curso del ler. grado. En estas investigaciones, Ios análisis de los datos se elaboraron en forma vertical. Analizamos integralmente cada entrevista y, a continuación, las agrupamos de forma que el desarrollo lógicocausal observado en la totalidad de las entrevistas quedase reflejado en los grupos que identificamos.
Los resultados obtenidos también fucron diferentes. Fn los primeros trabajos el énfasis se situó más en los modelos o patrones de representación, en una posicion de clasificación. Al cambiar el tipo de análisis de datos, logramos obtener un desarrollo psicogenético de los conceptos estudiados, lo que nos posibilitó entender cuáles son las etapas o estadios que cada individuo debe pasar en la construcción de su conocimiento, además de permitir la comprensión de cuáies son las diferencias y mecanismos de sucesión entre esas etapas.

En la segunda línea de investigación, con la cual intentamos comprobar en el aula metodologías de enseñanza, tenemos tres trabajos realizados (Anexo). En el análisis crítico de esos trabajos conviene discutir dos problemas: la construcción de las metodologías de enseñanza y el problema crucial que es la metodología de investigación para la compilación y análisis de datos.

Con relación a la metodología de enseñanza, las tres disertaciones contaron con las mismas bases teóricas: el desarrollo psicogenético de los conceptos a enseñar (fluctuación, proporción y velocidad angular), la historia de la ciencia y la teoría piagetiana como base de la organización social y sistemática del auła y como orientadora del papel del profesor a lo largo de la enseñanza ( $A$ bib 1988 y Silva 1990$)$.

Con respecto a la metodología de investigación que orienta la compilación y el análisis de los datos, las tres disertaciones difieren mucho y muestran la evolución técnico-metodológica del grupo.

En el primer trabajo sobre enseñanza de proporcionatidad, fechado de 1986 , pero que en realidad se planeó y ejecutó en 1983, usamos uno de los diseños de investigación de Campbell-Stanley (1979). Los datos se limitaron a la comparación estadística entre el grupo de control y el experimental en tres pruebas: preevaluación, post evaluación y retención. A pesar de que los datos presentaron ganancias estadísticamente significativas en todas las medidas, perdimos lo esencial del problema, que era la evolución de los alumnos, a consecuencia de las nueve actividades de enseñanza. En verdad, este aspecto sólo quedó claro mucho más tarde; en aquel momento sólo sentimos que algo se había perdido.

No usamos los grupos experimental y del control en lia segunda disertación (que versó sobre la enseñanza de la fluctuación). Bajo la influencia de imumerables investigaciones que destacaban la importancia de los estadios piagetianos en el entendimiento de Física (Chiapetta 1976, Lawson y Renner 1974 1975, Lawson y Sollman 1977, Rowell y Sawson 1977), focalizamos el análisis de nuestros datos en la relación entre el aprendizaje y el nivel de desarrollo medido por la escala de langeot (1974).

Fn esta investigación el análisis estadístico de tos datos tampoco nos satisfizo, pues obtuvimos lo esperado: los alumnos formales, con sus estructuras lógicas ya formadas, obtuvieron rendimientos superiores a Ios alumnos de los períodos concreto e intermediario. Estábamos en el mismo «impasse» que Duckworth (1979): «...o les 
enseñanmos demasiado pronto y ellos no pueden aprender, o demasiado tarde y ellos ya lo saben». Este dilema, como lo señaló la propia autora, es falso y se basa en la suposición de que cualquier preparación intelectual se fundamenta, únicamente, en las estructuras lógicas.

Regresamos a la investigación en el aula solamente en 1988 cuando, después de haber estudiado tex tos como el de Bovet et al. (s/f), nos sentimos más seguros sobre cómo analizar los datos de investigaciones que enfocasen la dinámica de la enseñanza. En la disertación de Silva, sobre la enseñanza de volocidad angular, intentamos averiguar si los procesos de desequilibrios cognoscitivos podían llevar al aprendizaje del concepto. A partir de la categorización de cuatro pruebas construidas tomando por base las contradicciones que habían aparecido en el estudio psicogenético, logramos entender la trayectoria de cada alumno y los cambios en su raciocinio a lo largo y después de las clases.

A pesar de que aún tenemos muchas preguntas sin respuesta, el tipo de análisis de datos desarrollado fue, sin lugar a duda, muy superior a los anteriores y pretendemos repetirlo en las próximas investigaciones.

\section{PLANES PARA EL FUTURO}

En los próximos años el grupo pretende continuar con las dos líneas presentadas: psicogénesis de los conceptos e investigaciones en el aula.

Estamos elaborando tres trabajos en Ia línea psicogenética. Intentamos averiguar el desarrollo del concepto geométrico de similitud, del concepto biológico de descomposición orgánica y del concepto físico del calor.

Para los trabajos en el aula la revisión que hicimos parece afirmar el papel de la interacción en el desarrolio del alumno y la necesidad de que atendamos a un conjunto mayor de factores desestructurantes en los procesos de enseñanza y de aprendizaje. Esto significa un desplazamiento hacia el eje interaccionista-constructivista de la epistemología piagetiana, una de nuestras bases teóricas.

Otro aspecto tratado en las investigaciones en el aula, se relaciona con la identidad en la metodología de enseñanza (Coll 1985, Rowell 1985/1989, Kamil y Devries 1989, Gil 1988) y en la metodología de investigación que será la misma que ya se estableció en el trabajo de Silva, añadida al análisis de vídeo de las clases.

Intentamos, además, reunir los trabajos en enseñanza de Física en el segundo grado en torno a un contenido, aceptando la sugerencia del Primer Encuentro Latinoamericano de Grupos de Investigación en Enseñanza de Física, el cual sugirió el tema «calor y temperatura». Se planifica, tambiến, una enseñanza de forma que podamos estudiarla bajo varios ángulos.

Detallamos a continuación una serie de cuestiones que pretendemos investigar en los trabajos que desarrollaremos a lo largo de los próximos años, agrupadas bajo los siguientes enfoques:

1. Papel de la historia de la ciencia en la enseñanza.

¿El uso explícito de la historia de la ciencia puede auxiliar efectivamente la enseñanza para:

- propiciar un refinamiento de los conceptos clasificados en un determinado contenido?

- posibilitar la reelaboración (o elaboración) de un modelo explicativo más cercano a aquél que se acepta científicamente?

¿Se puede identificar, evaluar o medir este auxilio para atribuir a este uso explícito de la historia de la ciencia un papel muy definido en la enseñanza?

¿Se puede identificar o elegir el mejor momento para utilizarlo?

¿Qué estrategias permiten explicitar la historia de la Física en cursos de segundo grado y son, al mismo tiempo, compatibles con la naturaleza y objetivos de esos cursos?

¿El enfoque histórico le resulta, por lo menos, interesante al alumno?

Investigaciones: «construcción del signo numérico» y "calor y temperatura».

2. Construcción, por el alumno, del concepto de situación de enseñanza.

¿Las actividades que planeamos con el auxilio de la investigación psicogenética y de la historia de la ciencia producirán conflictos cognoscitivos en los alumnos?

¿Esos conflictos desencadenarán los desequilibrios/reequilibrios necesarios a la construcción, por el alumno, del conocimiento pretendido?

¿Cuáles son las trayectorias cognoscitivas del alumno a lo largo de un curso?

Investigaciones: "construcción del signo numérico", "calor y temperatura» y "Ciencias en el primer grado».

\section{Resolución de problemas}

¿Cómo relacionar la cuestión de la resolución de problemas con todo el proceso de construcción del conocimiento científico?

¿Cuál es el papel que la resolución de problemas tiene en el proceso de aprendizaje?

Investigaciones: "calor y temperatura», "Física en el 3er.grado" y "Geometría».

\section{Organización social del aula}

¿Cuál es el papel del profesor en el desarrollo de condiciones optimas para la interacción constructivista entre el alumno y el objeto de conocimiento? 
¿Cuál es el papel de la interacción social, desarrollada a través de la actividad de enseñanza, en el proceso de construcción de conocimientos?

Investigaciones: «calor y temperatura», «construcción del signo numérico», «Física en el 3er. grado» «Geometría» y "Ciencias en el ler, grado".

\section{Contexto de la realidad escolar}

Las situaciones de aprendizaje son polifacéticas y ocurren en contextos culturales densos que extrapolan los límites del aula (Erickson 1978, Spindler 1988). Partiendo de ese presupuesto, investigaremos:

¿Hasta qué punto un observador puede contribuir a la mejor comprensión del proceso de interacción profesor/ alumno que se establece en el aula durante la realización de investigaciones en la cscuela?

¿Cómo los alumnos, los profesores, los miembros del cucrpo directivo y los padres ven la nueva propuesta de enseñanza que se investiga y qué valor le atribuyen?

\section{REFERENCIAS BIBLIOGRÁFICAS}

ABIB, M.L.V.S., 1988. Uma Abordayem Piagetiana para o Ensino de Flutuaçäo dos Corpos. Textos: Pesquisa para o Ensino de Ciências no. 2. Faculdade de Fducação, Universidade de São Paulo.

BOVET, M. et al. s/d. Causalité et apprentissage. Pré-print Université de Genève. Faculté de Psycologie e des Sicences de l'Lducation. Section de Psychologie, Ch 121, Genève 4.

CAMPBELI, D. y STANLEY, J., 1979. Delineamentos experimentais e quase-experimentais de pesquisa. Editora Pedagógica c Universitária e Editora da Universidade de São Paulo.

CHIAPPETTA, L.A., 1976. Review of Piagetian Studies Relevant to Science Instrution at Secundary and College Level, Science Education, 60(2), pp. 253-261.

COLJ, C., 1983. Las aportaciones de la psicología a la educación: cl caso de la teoría genética y de los aprendizajes escolares, en Psicología Genética y Aprendizajes Éscolares. (Siglo XXI de España Editores)

COI.I. C., 1985. Acción, Interacción y Construcción del Conocimiento en Situaciones Educativas, Anuario de Psicologia, 33(2).

DUCKWOR'TH, F., 1979. Either we're too Early and they can't Learn it or we're too late and they know in already: the dilemma of Applying Piaget's, Harvard Educational Review, 49, pp. 297-312.

ERICKSON, F., 1978. Qualitative methods in research on teaching, en Handbook of research on teaching, third edition. (MacMillan: N.Y.).
¿Cómo las eventuales manifestaciones de resistencia pueden comprometer los resultados deseados?

¿La estructura administrativa facilita o dificulta la introducción de innovaciones?

Investigación: "calor y temperatura».

Señalar estas cuestiones ya nos sitúa un paso más allá de los trabajos que hasta el momento hemos realizado. Elaborar métodos y procedimientos eficientes para atacarlas será el inicio de una «nueva equilibración», fundamental para que alcancemos, en la enseñanza, las mejoras que pretendemos.

\section{NOTAS}

(1) 1er. grado: equivale a EGB. $2^{\circ}$ grado: equivale a BUP.

(2) 1er. grado: abarca 8 cursos. $2^{2}$ grado: abarca 3 cursos.

GAGLIARDI, R., 1988. Cómo utilizar la Historia de las Ciencias en la Enseñanza de las Ciencias, Enseñanza de las. Ciencias, 6(3), pp. 29-296

GIL, D. et al., 1988. La resolución de problemas de lápiz y papel como activiojad de investigación, Investigación en la Escuela, 6, pp. $3-20$.

GOHAU, G., 1987. Difficultes d'une pedagogie de la découverte dans l'enseignement des sciences, Aster, 5, pp. 49-69.

KAMII, C. y DEVRIES, R., 1985. O Conhecimento Físico na Educaçấo Pré-Escolar. (Porto Alegre: Artes Médicas).

LACOMBF, G., 1987. Pour l'introduction de l'histoire des Sciences dans l'enseignement du Second Cycle, Aster, 5 , pp. 87-116.

LAWSON, A.E. y RFNNER, J.W, 1974. A Quantitative Analysis of Responses to Piagetian Tasks and I'ts Implications for Curriculum, Science Education, 58(4), pp. 545-559.

LAWSON, A.E., WOLLMAN, W. Y WARREN, T.P., 1975. Physics Problems and Process of Self Regulation, The Physics Teacher, vol. 13, no. 8, pp. 470-475.

LAWSON, A.E. y RENNER, J.W., 1975. Relationships of Science Subject Matter and Developmental Levels of Learns, Journal of Research in Science Teaching, 12(4), pp. 347-385.

PIAGET, J. y GARCÍA, R., 1982. Piscogénesis e Historia de la Ciencia. (Siglo XXI Editores: México).

ROWELL, J.A. y SAWSON, C.J., 1977. Teaching About floating an Sinking an Attempt to I.ink Cognitive Psycology 
with Classroom Practice, Science Education, 61(2), pp. 245 2.53.

ROWELL, J.A., 1985. Equilibration, Conflict and Instruction: A New Class Oriented Perspective, European Journal of Science Education, 7, pp. 331-344.

ROWELL, J.A., 1989. Piagetian Epistemology: Equitibration and the Teaching of Science, Syntese, 80, pp. 14I-162.
SILVA, D., 1990. O Ensino Construtivista da Velocidade Angular. Textos: Pesquisa para o Énsino de Ciências, n. 4 , Faculdade de Educação, Universidade de Sāo Paulo.

SPINDLER, G. (ed.), 1988. Doing the Ethnography of Schooling: Educational Antropology in Action. (Rinehart and Winston: N.Y.).
ANEXO

\section{RESLMEN DE TESIS PRESENTADAS POR EL GRUPO DE ENSFÑNANA DF CIENCIAS DE LA FACULDADE DE EDUCAÇĀO DE LA UNIVERSIDADE DE SÄO PAULO}

\section{INVESTIGACIONES EN PSICOGÉNESIS DE I,OS CONCEPTOS}

1. Estudio de las nociones espontáneas acerca de fenómenos relativos a la luz en alumnos de $11-18$ años, de $\mathrm{S}$. Krapaz Teixeira (1982)

Esta investigación intentó determinar los modelos de visión que nuestros alumnos presentan; trabajamos, además, con espejos y lentes.

2. Desarrollo del concepto de velocidad: un estudio a partir de problemas típicos de auła, de O.P.B. Teixeira (1985).

En esta investigación partimos de los trabajos y a realizados por el grupo de Piaget sobre el desarrollo del concepto de velocidad e intentamos estudiar la problemática del concepto de velosidad cuando éste se presenta en el lenguaje propio de la enseñanza de Física.

3. Resolución de problemas aritméticos simples abarcando adición y subtracción, por escolares de primer curso, de M... Boldrin (1987).

Intentamos detectar los raciocinios lógicos de los niños del primer curso de ler. grado cuando éstos necesitaban solucionir problemas de suma y resta. La investigación indicó que "a dificultad que los niños tienen en los problemas de substrac:ción se debe a que éstos requieren cuatro tipos diferentes de raciocinio para su resolución.

4. Desarrollo y aprendizaje del concepto de aceleración en adolescentes, de C.E. Laburú (1987).

En esta investigación intentamos describir los procedimiento; usados por los individuos (alumnos de 11 a 16 años) en su interpretación de la noción de aceleración. Detectamos que esta representación se une a otros conceptos físicos como fuerza, velocidad, posición, aumento de velocidad y variaciót: de velocidad. En el análisis de los resultados vimos que se podían agrupar los patrones de aceleración en dos grandes grupos determinados por la causalidad intrínseca referente a cada grupo, causalidad esta unida a las dificulta- des inherentes a la velocidad y otra unida a la concepción de fuerza.

5. La formación del concepto de cantidad de movimiento y su conservación, de A.M.P. de Carvalho (1986).

Procuramos entender cómo el niño y el adolescente (7 a 14 años) explican el fenómeno del choque mecánico. Como resultado de esta investigación obtuvimos algunos puntos fundamentales para la enseñanza de Ffsica en la escuela. Averiguamos que en las explicaciones de los fenómenos, los niños y adolescentes toman como necesaria la conservación del movimiento $y, a$ partir de ese hecho, se construye la noción de cantidad de movimiento ( «impulso» para los niños), así como su relación con la masa y velocidad. Constatamos, además, que en el desarrollo de las explicaciones causales de estos fenómenos (choques), los tres conceptos básicos de la dinámica (f, p, l) aparecen diferenciados.

6. Un estudio psicogenético de la velocidad angular y la construcción de su enseñanza, de Dirceu da Silva (1988).

En la primera parte de esta investigación estudiamos el desarrollo psicogenético del concepto de velocidad angular en niños y adolescentes de 7 a 14 años. Averiguamos que los individuos construyen espontáneamente la idea de velocidad angular, pero siempre la asocian a la idea de velocidad lineal, a través de la solución o equilibración de una contradicción aparente entre las dos velocidades. Esa contradicción se equilibra en el momento en que la idea de velocidad deja de ser única y aparece, así, la velocidad angular. La segunda parte de la investigación, que es la construcción de la enserianza, se desarrollará más adelante.

7. Conservación y modelo corpuscular; un estudio transversal de las explicaciones de los estudiantes para transformaciones de la materia, de I. Trivelato (1989).

El objetivo de este trabajo fue el estudio de la cvolución de las nociones del modelo corpuscular de la materia y cómo ellas se relacionan con el desarrollo de las nociones de conservación de las cantidades físicas. Entrevistamos cuarenta y cuatro estudiantes de los cuatro áltimos cursos del ler. grado y de los dos primeros cursos de la carrera universitaria, y registramos las explicaciones que ellos nos dieron a dos transformaciones que abarcaron variaciones en la concentración de materia. 
8. Estudio psicogenético de la noción de centro de masa: una contribución a la enseñanza de Física, de M.R. do Valte Fitho (1989).

Entrevistamos, en esta investigación, a cuarenta y dos individuos de edades entre 6 y 16 años. Utilizamos cinco situaciones experimentales que abarcaban cucrpos en equilibrio, en movimiento de translacion y en movimiento de rotación. Los resultatos indicaron que se pueden clasificar las nociones utilizadas por los individuos en tres grandes grupos jerarquizados, señalando una evolución y considerando que el desarrollo de las nociones se caracteriza globalmente por la percepción de simetrías, por la coordinación entre el todo y la parte, por la posibilidad de reflexionar en términos de compensación.

9. La construcción de un conocimiento en una actividad en grupo, de I. Maccione (1989).

Esta investigación se diferencia un poco de las anteriores al no ser un estudio de desarrollo psicogenético. Pretendimos, en este trabajo, averiguar cómo los alumnos, en una interacción social de grupo, pueden llegar a construir un concepto-fuerza centripeta. Observamos el trabajo de seis grupos de alumnos del primer curso del segundo grado. Analizamos la construcción del conocimiento físico y del conocimiento lógico-matemático elaborado en las discusiones de los grtipos.

\section{Un estudio psicogenético de ideas que evolucionan hacia la} noción de campo, de R. Nardi (1990).

Esta investigación intentó señalar las explicaciones causales dadas por cuarenta y cinco individuos de 6 a 17 años sobre cinco situaciones desencadenadoras, abarcando el concepto de campo de fuerza. Los datos emergentes de las entrevistas clínicas permiticron, además, averiguar la evolución de la concepción de la l'terra como cuerpo cósmico, la evolución del léxico científico y la influencia dei modelo de campo transmitido por televisión a los individuos a través de dibujos animados.

\section{INVESTIGACIONES EN ENSEŃANZA}

1. Enscñanza del concepto de proporcionalidad, de Adriano R. Ruiz. (1986).

En esta investigación pusimos a prueba una metodología para la cnseñanza de proporcionalidad considerando el hecho de queel raciocinio proporcional abarcauna estructura de pensamiento muy compleja. Con esa preocupación, desarrollamos nueve actividades de enseñanza, en todas utilizando material concreto. Hillas llevaron a los alumnos a que pasaran de un concepto únicamente cualitativo al establecimiento de una relación cuantitativa, permitiendo que surgiera del propio trabajo del alumno la necesidad de explotación de situaciones que le posibilitarían identificar la proporcionalidad como una relación de razón constante.

L. a metodología de investigación fue el modelo que abarea un grupo de control y un grupo experimental. Los grupos, de control y experimental, se constituyeron con los alumnos de dos aulas de séptimo curso de escuclas públicas. Las medidas fueron la preevaluacion, la postevaluación y la prueba de retención.

Hubo una ganancia estadísticamente significativa (nivel de $0,05)$ por parte de los componentes del grupo experimental, tanto si se considera la prueba integraimente como si se consideran sus partes: no-numéricas y numéricas. Además de que las ganancias medianas son estadísticamente significativas, destacamos el hecho de que la gran mayoría de los alumnos $(82 \%)$ concentr6 sus aciertos entre el $70 \%$ y el $100 \%$ de Ios problemas solucionados correctamente.

2. La interferencia del nivel de desarrollo cognoscitivo en el aprendizaje de un contenido de Física, de M.L.V. Santos Abib (1983)

La base teórica de esta investigación fue el trabajo de Inhelder y Piaget, en el cual los autores muestran detalladamente la construcción conceptual y el raciocinio lógico-matemático que utilizan los individuos al solucionar el problema de fluctuación de los cuerpos.

Así, cuando planificamos nuestra metodología de cnseñanza, intentamos incluir actividades que: 1) permiticran explicitar las concepciones de los alumnos sobre el fenómeno, con posibilidad de elaboración y prueba experimental de las hipótesis que, en general, los alumnos señalan sobre las causas de la fluctuacion, con oportunidad de utilizar el raciocinio hipotetico-deductivo; 2) propiciasen el uso del método de control de variables y de las medidas de masa, volumen y densidad de objetos y de líquidos, de modo que permitieran la claboración de las nociones de las magnitudes relevantes; 3) eviden. ciasen el raciocinio proporcional, necesario a la comprensión de densidad, tanto en los momentos de actividad experimental, como en las discusiones y resoluciones de ejercicios y problemas. Para lograr tales objetivos organizamos la enseñanza en una serie de problemas experimentales solucionados en grupo por los alumnos, y que ofrecieron la oportunidad para que construyeran su conocimiento en un proceso activo, en interacción con el objeto experimental, además de resolver en cada problema el conflicto cognoscitivo que se establecía entre la previsión, es decir, el establecimiento de las hipótesis y la observación.

3. Un estudio psicogenético de la velocidad angular y la construcción de su enseñanza, de Dirceu da Silva (1988).

Fn esta segunda parte utilizamos las informaciones que obtuvimos en el estudio psicogenético del concepto en cuestión (brevemente descrito en el apartado 6 de este anexo). Aplicamos en la primera clase una preevaluación basada en las situationesproblema y categorizamos las ideas previas de Ios alumnos que revelaron la precariedad de las explicaciones sobre las velocidades en fenómenos que abarcaban rotación. En cl aula intentamos llevar a los alumnos a que explicitaran sus ideas y a que interactuaran cognoscitivamente entre ellos a traves de discusiones en grupo, para reelaborar sus concepciones. Intentábamos, así, líegar a una situación de desequilibrio cognoscitivo. En ese momento, presentamos las dos velocidadescomonecesarias a la explicación de los fenómenos utilizando la historia de la ciencia. Se siguieron dos clases de resolución de ejercicios del libro-texto usado. Se aplicaron una postevaluación dos semanas despues de la ensenanza y dos pruebas de retención (la primera nueve semanas después de la postevaluación y la segunda veinticinco semanas despues de la ensenanza). Hificimos la categorización y clasificación de los alumnos después de cada prueba. Nuestros resultados fueron excelentes ya que, con básicamente cuatro clases, llegamos al nivel de aprenłtizaje totalmente satisfactorio de $85 \%$ de los alumnos. Obtuvimos muchos casos de oscilaciones entre categorias, loque confirma que los procesos de desequilibración y equilibración cognoscitiva dependen de cada individuo con ritmos y momentos propios para que puedan ocurrir. 